\title{
Probing Nitride Thin Films in 3-Dimensions using a Variable Energy Electron Beam
}

Carol Trager-Cowan ${ }^{1}$, D. McColl ${ }^{1}$, F. Sweeney ${ }^{1}$, S. T. F. Grimson ${ }^{1}$, J-F. Treguer ${ }^{1}$, A. Mohammed $^{1}$, P. G. Middleton ${ }^{1}$, S. K. Manson-Smith ${ }^{1}$, K. P. O'Donnell ${ }^{1}$, W. Van der Stricht $^{2}$, I. Moerman ${ }^{2}$ and P. Demeester ${ }^{2}$, M. F. Wu ${ }^{3}$, A. Vantomme ${ }^{3}$, D. Zubia ${ }^{4}$ and S. D. Hersee ${ }^{4}$

${ }^{1}$ Dept. Physics and Applied Physics, University of Strathclyde, Glasgow G4 0NG, UK.

${ }^{2}$ IMEC-INTEC, University of Gent, Gent 9000, Belgium.

${ }^{3}$ KULeuven, Leuven, Belgium.

${ }^{4}$ University of New Mexico, Center for High Technology Materials, 1313 Goddard, SE

Albuquerque, NM, USA.

\section{ABSTRACT}

In this paper we illustrate the application of electron beam techniques to the measurement of strain, defect and alloy concentrations in nitride thin films. We present brief comparative studies of CL spectra of $\mathrm{AlGaN}$ and InGaN epilayers and EBSD patterns obtained from two silicon-doped $3 \mu \mathrm{m}$ thick GaN epilayers grown on an on-axis (0001) sapphire substrate and a sapphire substrate misoriented by $10^{\circ}$ toward the m-plane (10 10$)$.

\section{INTRODUCTION}

Thin films incorporating GaN, InGaN and AlGaN are presently arousing considerable excitement because of their suitability for UV and visible light emitting diodes and laser diodes. However, the films are of variable quality because of the lattice mismatch between them and presently used substrates (sapphire and $\alpha-\mathrm{SiC}$ ). We are presently using a number of electron beam analysis techniques namely cathodoluminescence (CL) imaging, CL spectroscopy and electron backscattered diffraction (EBSD), to investigate both the structural and optical properties of nitride films in 3-dimensions. Information in the $3^{\text {rd }}$ dimension is extracted by acquiring data at different electron beam energies. Electron beams of energy between 1 and $20 \mathrm{keV}$ are well matched to the length scales typical of nitride heterostructures. For example, a 2 $\mathrm{keV}$ beam deposits energy to a depth of $\approx 50 \mathrm{~nm}$, a $10 \mathrm{keV}$ electron beam deposits energy to a depth of $\approx 600 \mathrm{~nm}$, while a $20 \mathrm{keV}$ beam deposits energy to a depth of $\approx 2 \mu \mathrm{m}$ in a GaN layer.

In this paper we illustrate the application of electron beam techniques to the analysis of nitride thin films by presenting brief comparative studies of

(i) $\quad \mathrm{CL}$ spectra of $\mathrm{AlGaN}$ and InGaN epilayers

and

(ii) EBSD patterns obtained from two silicon-doped $3 \mu \mathrm{m}$ thick $\mathrm{GaN}$ epilayers grown on an on-axis (0001) sapphire substrate and a sapphire substrate misoriented by $10^{\circ}$ toward the m-plane $(10 \overline{1} 0)$. 


\section{EXPERIMENTAL DETAILS}

All of the samples described in this paper were grown by metalorganic vapour phase epitaxy (MOVPE) on (0001) sapphire substrates (unless otherwise indicated). CL spectra were acquired using a home-built electron beam excitation system providing beams of energy up to $30 \mathrm{keV}$ in a spot size of $\approx 200 \mu \mathrm{m}$ at current densities up to $20 \mathrm{~A}$ $\mathrm{cm}^{-2}$ (but limited in the work to be described here to $<10 \mathrm{~mA} \mathrm{~cm}$ ). In the present work the front faces of the samples were positioned normal to the exciting electron beam and CL detected from the sample edge. Samples were cooled to a temperature of approximately $25 \mathrm{~K}$ using a closed cycle helium cryorefrigerator. Spectra were acquired using an Oriel InstaSpec ${ }^{\mathrm{TM}}$ cooled 2-dimensional CCD array mounted at the output focal plane of a Chromex $0.5 \mathrm{M}$ monochromator.

A Cambridge 600S scanning electron microscope has been adapted by the addition of a home-built CL detection system (incorporating an Oxford Instruments parabolic mirror light collector) and a home-built EBSD imaging system. These allow the acquisition of comparative physical and luminescent images and EBSD patterns from the same area of a sample.

\section{EXPERIMENTAL RESULTS AND DISCUSSION}

\section{CL profiling of AlGaN and InGaN epilayer}

A variable energy electron beam has been used to investigate an AlGaN-on-GaN bilayer, grown on a sapphire substrate. The thickness of the $\mathrm{AlGaN}$ and $\mathrm{GaN}$ layers were

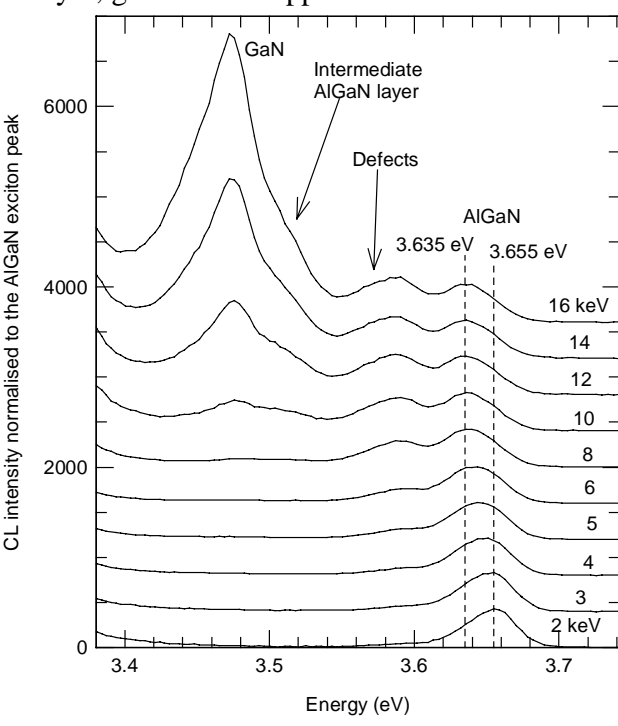

Figure 1. CL spectra acquired at different electron beam energies from an AlGaN/GaN/Sapphire structure. determined by Rutherford

backscattering (RBS) to be $0.48 \mu \mathrm{m}$ and $0.44 \mu \mathrm{m}$ respectively, RBS measurements also revealed the mean aluminium concentration to be $7 \%$.

Figure 1 shows low temperature CL spectra acquired from the $\mathrm{AlGaN} / \mathrm{GaN} /$ sapphire layers at electron beam energies from $2 \mathrm{keV}$ to $16 \mathrm{keV}$. Spectra have been normalised in intensity and shifted vertically for clarity. It can be seen that the highest energy CL emission band, which we attribute to bound exciton emission from the AlGaN, red shifts from $3.655 \mathrm{eV}$ to $3.635 \mathrm{eV}$ as the electron beam energy increases from $2 \mathrm{keV}$ to 16 $\mathrm{keV}$. No beam current dependence of this peak shift is observed. At electron beam energies $\geq 3 \mathrm{keV}$ we observe a band peaking at $\approx 3.59$ 
$\mathrm{eV}$. This band increases in intensity with respect to the AlGaN bound exciton band as the electron beam energy increases. At electron beam energies $\geq 8 \mathrm{keV}$, an emission band at $\approx 3.475 \mathrm{eV}$ becomes evident. We attribute this band to bound exciton emission from the underlying GaN epilayer. Also appearing at energies $\geq 8 \mathrm{keV}$ is a shoulder on the high energy side of the GaN bound exciton band at $\approx 3.52 \mathrm{eV}$.

In order to interpret these results, it is necessary to know to what depth an electron beam of given energy penetrates the structure. Monte Carlo simulations of the electron trajectories [1] in an $\mathrm{Al}_{0.07} \mathrm{Ga}_{93} \mathrm{~N}$ layer, were therefore carried out. Figure 2 shows the results of these simulations.

Figure 2 illustrates that the electron beam penetrates deeper into the structure as its

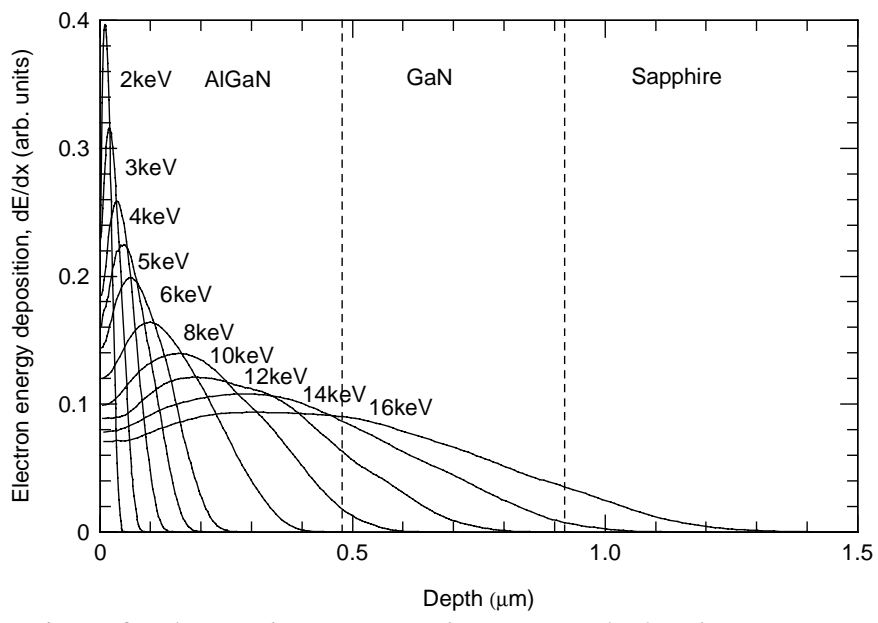

Figure 2. Electron beam energy deposition calculated using Monte-Carlo simulations of the electron trajectories in an $\mathrm{Al}_{0.07} \mathrm{Ga}_{0.93} \mathrm{~N}$. layer.

energy increases. It implies that electron beams with energy of $2 \mathrm{keV}$ to $6 \mathrm{keV}$ will address only the $\mathrm{Al}_{0.07} \mathrm{Ga}_{93} \mathrm{~N}$ layer, while electron beams with incident energy $\geq 8 \mathrm{keV}$ break through into the GaN underlying layer while electron beams with energy $\geq 14 \mathrm{keV}$ penetrate the sapphire substrate. This explains why electron beam energies $\geq 8 \mathrm{keV}$ are required to excite significant emission from the $\mathrm{GaN}$ underlying layer. Some emission is just discernible from the GaN layer at electron beam energies $\leq 8 \mathrm{keV}$, we attribute this to secondary excitation of the $\mathrm{GaN}$ by $\mathrm{CL}$ emission from the AlGaN layer.

With increasing energy, the electron beam will excite an increasing proportion of material near and at the interface between the AlGaN and underlying GaN layer. We might expect to observe emission associated with defects due to this interface. We attribute the peak observed at $\approx 3.59 \mathrm{eV}$ to such defect emission, the intensity of this emission increasing with increasing electron beam energy as the electron beam excites higher proportions of defected material.

We propose that the shoulder on the high energy side of the $\mathrm{GaN}$ bound exciton band at $\approx 3.52 \mathrm{eV}$ is due to an AlGaN layer with low aluminium concentration $(\approx 2 \%)$ [2] at the interface between the $\mathrm{AlGaN}$ and $\mathrm{GaN}$ layers. We base this conclusion on the fact that this shoulder appears at the same electron beam energy as the GaN bound exciton 
band and must therefore occur at a similar depth in the sample as the top of the GaN layer. The intensity of the shoulder while similar to that of the GaN bound exciton band at $8 \mathrm{keV}$, drops to less than half the intensity of the GaN emission band at $16 \mathrm{keV}$. This reduction in relative intensity of the shoulder may be due to the absence of the source of this emission as the electron beam probes deeper into the sample, i.e., the shoulder emission at $\approx 3.52 \mathrm{eV}$ is due to a thin sandwich layer.

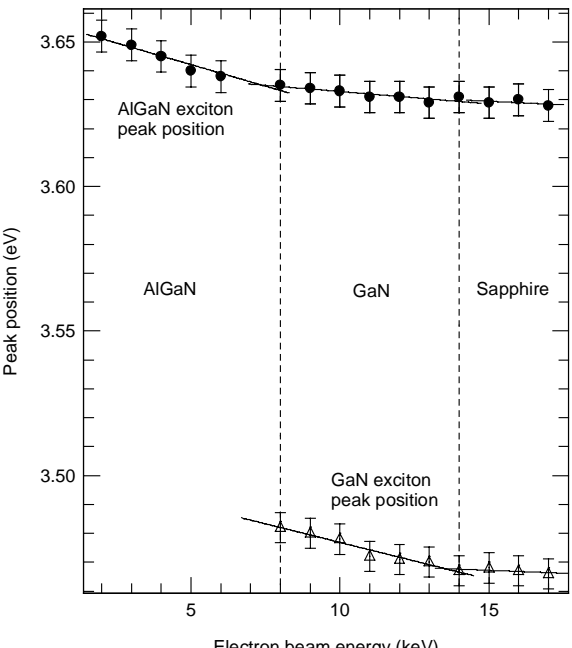

Figure 3. Shift of the peak of the AlGaN and GaN exciton peaks as a function of electron beam energy.
To understand the origin of the observed red shift of the AlGaN bound exciton band with increasing energy, we plot its peak position as a function of electron beam energy in figure 3 . We also plot the dependence of peak position of the GaN bound exciton band. Figure 3 shows that the peak of the $\mathrm{GaN}$ bound exciton band shifts to the red by $15 \mathrm{meV}$ as the electron beam energy is increased from 8 to $14 \mathrm{keV}$. These electron beam energies correspond to penetration depths of approximately $480 \mathrm{~nm}$ and $1.2 \mu \mathrm{m}$ respectively, i.e., from the approximate location of the $\mathrm{AlGaN} / \mathrm{GaN}$ interface to the GaN/sapphire substrate interface. We attribute this red shift to a relaxation towards the sapphire substrate of compressive strain in the $\mathrm{GaN}$ layer, where the strain is due to the overlying AlGaN layer. From the work of Kim et al [3] and Edwards et al [4], we estimate the change in stress across the GaN layer to be of order $4 \mathrm{kbar}$, this corresponds to a change in strain of approximately $0.15 \%$. The $\mathrm{AlGaN}$ peak is seen to red shift by $20 \mathrm{meV}$ as the electron beam energy is increased from 2 to $8 \mathrm{keV}$. These electron beam energies correspond to the electron beam penetrating from $50 \mathrm{~nm}$ to $480 \mathrm{~nm}$ into the layer, i.e., from close to the surface of the AlGaN layer to the $\mathrm{AlGaN} / \mathrm{GaN}$ interface. As the electron beam energy increases from $8 \mathrm{keV}$ to $16 \mathrm{keV}$ the AlGaN peak continues to red shift as energy is deposited deeper into the sample. A total shift of $22 \mathrm{meV}$ is observed. In this case there is more than one possible explanation for the red shift of the peak. It may be attributed to the relaxation towards the surface of the $\mathrm{AlGaN}$ layer of the tensile strain to which the underlying GaN subjects the AlGaN layer. Alternatively, the shift may be due to an increase in the aluminium concentration. A red shift of $22 \mathrm{meV}$ from $\approx 3.65 \mathrm{eV}$ corresponds to a change in aluminium mole fraction from $\approx 8 \%$ at the surface of the sample to $\approx 7 \%$ at the interface between the $\mathrm{AlGaN}$ and $\mathrm{GaN}$ epilayers [2]. It could be also due to a combination of a variation of strain and aluminium concentration. However, without an independent method of measuring either the strain or the aluminium concentration with a resolution of order 10 nm we cannot come to a definitive conclusion.

A variable energy electron beam has also been used to investigate an InGaN epilayer $(\approx 400 \mathrm{~nm}$ thick) grown on an epilayer of $\mathrm{GaN}(1.0 \mu \mathrm{m})$, which in turn was grown on a sapphire substrate [5]. Figure 4 shows the peak position of the InGaN 


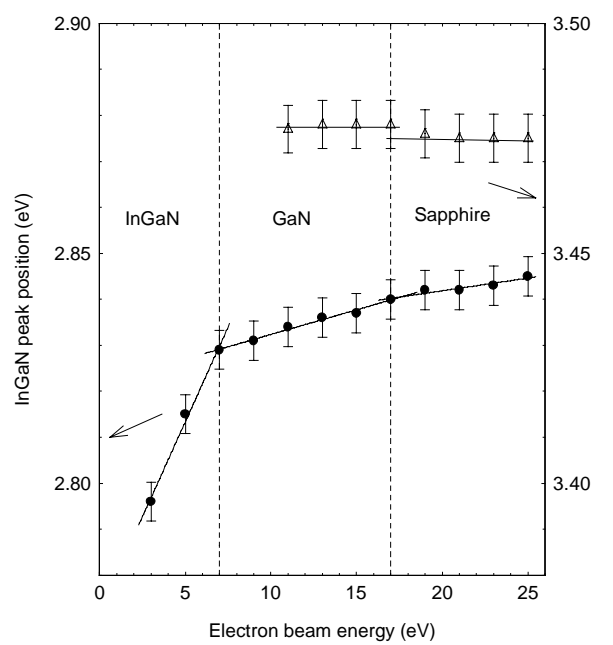

Figure 4. Shift of the peak of the InGaN and GaN emission peaks as a function of electron beam energy emission band as a function of electron beam energy. We also plot the dependence of peak position of the GaN emission band. In this case there is no significant shift of the $\mathrm{GaN}$ peak position therefore no change in strain is detectable. The InGaN peak is seen to blue shift by $33 \mathrm{meV}$ as the electron beam energy is increased from 3 to 7 $\mathrm{keV}$. No beam current dependence of this peak shift is observed. These electron beam energies correspond to the electron beam penetrating from $90 \mathrm{~nm}$ to $350 \mathrm{~nm}$ into the layer, i.e., from close to the surface of the InGaN layer to the neighbourhood of the InGaN/GaN interface. As the electron beam energy increases from $7 \mathrm{keV}$ to 25 $\mathrm{keV}$ the InGaN peak continues to blue shift as more energy is

deposited deeper into the sample. A total shift of $50 \mathrm{meV}$ is observed. As for the AlGaN epilayer discussed previously, there is more than one possible explanation for the observed blue shift. It may be attributed to the relaxation towards the surface of the InGaN layer of the compressive strain to which the underlying GaN subjects the InGaN layer. Alternatively, the shift may be due to an increase in the indium concentration towards the surface of the epilayer. A blue shift of $50 \mathrm{meV}$ from $\approx 2.8 \mathrm{eV}$ corresponds to a change in indium mole fraction from $\approx 15 \%$ at the surface of the sample to $\approx 14 \%$ at the interface between the InGaN and $\mathrm{GaN}$ epilayers [6]. We have revised our values for the indium mole fraction from those published in [5] in line with the results discussed in [6]. The shift could also be due to a combination of a variation of strain and indium concentration. In this case we suggest that a change in indium concentration may indeed play a role. In [5] we attributed the shift to the "compositional pulling effect" as reported by Shimuzu et al (1997) [7] and Hiramatsu et al (1997) [8]. That is the indium mole fraction is observed to be low during the initial stages of InGaN growth on GaN epilayers, but increases with increasing film thickness. Again, as previously discussed for the AlGaN epilayer, without an independent method for measuring either the strain or the indium concentration with a resolution of order $10 \mathrm{~nm}$ we cannot come to a definitive conclusion as to the origin of the shift of the InGaN peak.

\section{EBSD patterns from GaN epilayers grown on misoriented sapphire substrates}

EBSD is a diffraction technique that allows crystallographic information to be extracted from samples in a scanning electron microscope [9]. In particular it allows the measurement of strain with a lateral spatial resolution of order $20 \mathrm{~nm}$ [10]. We present here some preliminary measurements from two silicon-doped $3 \mu \mathrm{m}$ thick GaN epilayers 
grown on an on-axis (0001) sapphire substrate and a sapphire substrate misoriented by

$10^{\circ}$ toward the m-plane $(10 \overline{1} 0)$. Figures 5 (a) and (b) show the EBSD patterns from the on-axis $\mathrm{GaN}$ epilayer and the $10^{\circ}$ epilayer respectively.

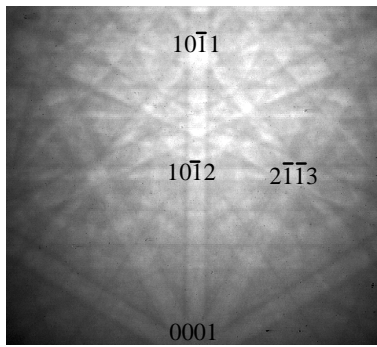

(a)

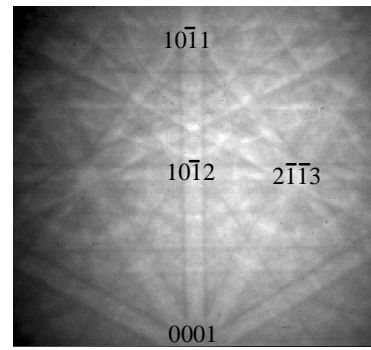

(b)

Figure 5. EBSD patterns from GaN epilayers grown (a) on and (b) $10^{\circ}$ off-axis respectively.

A compression of the pattern towards the [1011] zone axis (pole) from the [0001] zone axis is observed in figure 5 (a) when compared to figure 5 (b). We tentatively attribute this shift to a difference of the in-plane compressive strain between the 2 samples where the $10^{\circ}$ epilayer is under less compressive strain than the on-axis epilayer [11]. This result is consistent with those reported by Edwards et al [4] that GaN epilayers grown on vicinal substrates are less strained.

In the future we plan to quantify strain measurements using the EBSD technique. This will enable us to map the strain in GaN epilayers and will also allow us to make independent measurements of the strain in alloy samples such as the InGaN and AlGaN epilayers described in this paper. We may therefore come to definitive conclusions as to the origin of the observed shifts in the CL emission bands from these materials.

\section{REFERENCES}

1. E. Napchan and D B Holt, Inst. Phys. Conf. Ser. 87, eds A. G. Cullis et al., (IOP, 1987) pp. 733.

2. G. Steude et al, MRS Internet J. Nitride Semicond. Res. 4S1, G3.26 (1999).

3. S. Kim, et al, Appl. Phys. Lett., 67380 (1995).

4. N. V. Edward et al, Appl. Phys. Lett., 732808 (1998).

5. C. Trager-Cowan, et al, Mat. Res. Soc. Symp. Proc., 482, 715 (1998).

6. K. P. O'Donnell et al, this conference reference number W11.26

7. M. Shimuzu et al, Sol. St. Electr., 41145 (1997).

8. K. Hiramatsu et al, MRS Internet J. Nitride Semicond. Res., 26 (1997).

9. D. J. Dingley et al, Atlas of Backscattering Kikuchi Diffraction Patterns, (IOP, 1995).

10. D. J. Dingley and D. P. Field, Materials Science and Technology, 1369 (1997).

11. K. Z. Troost et al, Appl. Phys. Lett., 621110 (1993). 